\title{
ON LINEAR COMBINATIONS OF QUADRATIC FORMS
}

\section{LLOYD L. DINES}

The characteristics of linear combinations $\sum \lambda_{i} Q_{i}(x)$ of a given set of real quadratic forms

$$
Q_{i}(x) \equiv \sum_{k, l=1}^{n} a_{k l}^{i} x_{k} x_{l}, \quad i=1,2, \cdots, m,
$$

have been considered in several recent papers. ${ }^{1}$

One of the theorems in my earlier paper may be stated as follows:

$A$ necessary and sufficient condition that there exist a linear combination $\sum \lambda_{i} Q_{i}(x)$ which is positive definite is that there exist no set of points $x^{j}=\left(x_{1}^{j}, x_{2}^{j}, \cdots, x_{n}^{j}\right) \neq(0, \cdots, 0)(j=1,2, \cdots, r)$ such that

$$
\sum_{j=1}^{r} \mu_{j} Q_{i}\left(x^{j}\right)=0, \quad i=1,2, \cdots, m,
$$

the coefficients $\mu_{j}$ being positive.

Shortly after the publication of this paper, Fritz John kindly called my attention to the fact that a closely related result is contained in an earlier paper of his. ${ }^{2}$

Certainly John's paper contains essentially the "sufficiency" half of the theorem quoted above. Furthermore it introduces a very interesting suggestion in noting that the validity of relations (2) implies the existence of a quadratic form

$$
B(x) \equiv \sum_{k, l=1}^{n} b_{k l} x_{k} x_{l}
$$

which is definite or semi-definite, and such that

$$
\sum_{k, l=1}^{n} a_{k l}^{i} \cdot b_{k l}=0, \quad i=1,2, \cdots, m .
$$

Presented to the Society, February 27, 1943; received by the editors August 31, 1942.

${ }^{1}$ Finsler, Über das Vorkommen definiter und semidefiniter Formen in Scharen quadratischer Formen, Comment. Math. Helv. vol. 9 (1937) pp. 188-192. Hestenes and McShane, $A$ theorem on quadratic forms and its application in the calculus of variations, Trans. Amer. Math. Soc. vol. 47 (1940) pp. 501-512. Dines, On the mapping of $n$ quadratic forms, Bull. Amer. Math. Soc. vol. 48 (1942) pp. 467-471.

${ }^{2} A$ note on the maximum principle for elliptic differential equations, Bull. Amer. Math. Soc. vol. 44 (1938) pp. 268-271. 
This condition, unlike those occurring in other treatments of the problem, focuses attention on the coefficients of the given forms. Because of its purely algebraic character and its possible usefulness in applications it seems worthy of further study, and that is the purpose of the present note. In Theorem I there is an analysis of possibilities in terms of this relationship, and in Theorem II there is an equivalent statement from a different and possibly interesting point of view.

1. Orthogonality of quadratic forms as to their coefficients. The bilinear form consisting of the sum of products of corresponding coefficients of two quadratic forms

$$
Q(x) \equiv \sum_{k, l=1}^{n} a_{k l} x_{k} x_{l}, \quad B(x) \equiv \sum_{k, l=1}^{n} b_{k l} x_{k} x_{l}, \quad a_{k l}=a_{l k}, b_{k l}=b_{l k},
$$

will be denoted by $(Q \cdot B)$. That is ${ }^{3}$

$$
(Q \cdot B)=\sum_{k, l=1}^{n} a_{k l} \cdot b_{k l}
$$

Obviously $(Q \cdot B)=(B \cdot Q)$; and for a set of forms such as those in (1), $\left(\sum \lambda_{i} Q_{i} \cdot B\right)=\sum \lambda_{i}\left(Q_{i} \cdot B\right)$. Also it may be easily verified that $(Q \cdot B)$ is invariant under the group of orthogonal transformations on $x_{1}, x_{2}, \cdots, x_{n}$.

If the two quadratic forms $Q(x)$ and $B(x)$ satisfy the relation $(Q \cdot B)=0$ they will be said to be orthogonal as to coefficients (or c-orthogonal).4

Relative to a given system of real quadratic forms (1), we now state

Theorem I. (a) A necessary and sufficient condition that there exist a definite linear combination $\sum \lambda_{i} Q_{i}$ is that every quadratic form c-orthogonal to all the $Q_{i}$ be indefinite.

(b) A necessary and sufficient condition that every linear combination $\sum \lambda_{i} Q_{i}$ be indefinite is that there exist a definite quadratic form c-orthogonal to all the $Q_{i}$.

(c) A necessary and sufficient condition that there exist a semi-definite

${ }^{3}$ M. R. Hestenes has kindly called attention to the fact that this function of the coefficients of two forms was used by L. Fejér in a paper entitled Über die Eindeutigkeit der Lösung der linearen partiellen Differentialgleichung zweiter Ordnung, Math. Zeit. vol. 1 (1918) pp. 70-73. Fejér obtained a theorem which may be stated in our notation as follows: If $Q(x)$ and $B(x)$ are both non-negative, then $(Q \cdot B) \geqq 0$.

${ }^{4}$ However, to avoid bothersome trivialities, we shall assume that an assertion $(Q \cdot B)=0$ implies per se that each of the forms $Q \cdot B$ has at least one nonzero coefficient. In the same spirit, the notation $\sum \lambda_{i} Q_{i}$ implies per se that at least one $\lambda_{i}$ is different from zero. 
(but no definite) linear combination $\sum \lambda_{i} Q_{i}$ is that there exist a semidefinite (but no definite) quadratic form c-orthogonal to all the $Q_{i}$.

2. Proof of Theorem I(a). In view of the theorem quoted in our introduction, the validity of $\mathrm{I}(\mathrm{a})$ is an immediate consequence of the following lemma.

LEMma. A necessary and sufficient condition that the quadratic forms (1) admit relations (2) is that there exist a definite (or semi-definite) form $B(x)$ such that

$$
\left(Q_{i} \cdot B\right)=0, \quad i=1,2, \cdots, m .
$$

The first half of this lemma is due to John, as is the following neat proof of it.

Suppose there exist relations (2). These may be written

or

$$
\sum_{j=1}^{r} \mu_{j} \sum_{k, l=1}^{n} a_{k l}^{i} x_{k}^{j} x_{l}^{j}=0, \quad i=1,2, \cdots, m,
$$

$$
\sum_{k, l=1}^{n} a_{k l}^{i} \cdot b_{k l}=0 \text { where } b_{k l}=\sum_{j=1}^{r} \mu_{j} x_{k}^{j} x_{l}^{j} .
$$

Hence the quadratic form $B(x)$ with coefficients $b_{k l}$ is $c$-orthogonal to every $Q_{i}(x)$.

Furthermore the form $B(x)$ is definite or semi-definite, since

$$
B(x) \equiv \sum_{k, l=1}^{n} b_{k l} x_{k} x_{l}=\sum_{k, l=1}^{n}\left(\sum_{j=1}^{r} \mu_{j} x_{k}^{j} x_{l}^{j}\right) x_{k} x_{l}=\sum_{j=1}^{r} \mu_{j}\left(\sum_{k=1}^{n} x_{k}^{j} x_{k}\right)^{2} .
$$

Conversely, suppose there is a definite (or semi-definite) form $B$ satisfying (3). From the assumption of definiteness, it can be expressed in the form

$$
B(x) \equiv \sum_{k, l=1}^{n} b_{k l} x_{k} x_{l}=\sum_{j=1}^{r} \mu_{j}\left(\sum_{k=1}^{n} \alpha_{k}^{j} x_{k}\right)^{2}
$$

where the coefficients $\alpha_{k}^{j}$ are suitably chosen constants and the $\mu_{\text {, }}$ are positive constants. Hence

And in view of (3)

$$
b_{k l}=\sum_{j=1}^{r} \mu_{j} \alpha_{k}^{j} \alpha_{l}^{j}
$$

and equivalently

$$
\sum_{k, l=1}^{n} a_{k l}^{i} \sum_{j=1}^{r} \mu_{j} \alpha_{k}^{j} \alpha_{l}^{j}=0, \quad i=1,2, \cdots, m
$$




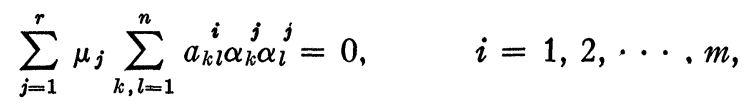

whence follows (2) with $x_{k}^{j}=\alpha_{k}^{j}$.

3. Proof of Theorem I(b). This proof is facilitated by the following corollary.

COROLlARY OF I(a). If two quadratic forms are c-orthogonal and one is definite, then the other is indefinite.

The sufficiency part of I(b) follows immediately. For if a definite form $B$ is $c$-orthogonal to every $Q_{i}$ it is $c$-orthogonal to every linear combination $\sum \lambda_{i} Q_{i}$, and hence by the corollary every such linear combination is indefinite.

To prove the riecessity part of $\mathrm{I}(\mathrm{b})$, we assume that every linear combination $\sum \lambda_{i} Q_{i}(x)$ changes sign. On account of the homogeneity property $Q(t x)=t^{2} Q(x)$, we are indeed justified in assuming that every such linear combination changes sign as $x$ varies on the unit hypersphere $\|x\|=1$ in the $n$-dimensional $x$-space.

The set of points $\mathfrak{M}_{1}$ with coordinates represented by

$\mathfrak{M}_{1}$ :

$$
\left(Q_{1}(x), Q_{2}(x), \cdots, Q_{m}(x)\right), \quad\|x\|=1,
$$

in $m$-dimensional space is closed and bounded. Hence, in view of our assumption, its convex extension $C\left(\mathfrak{M}_{1}\right)$ contains the origin of that $m$-dimensional space as an inner point. ${ }^{5}$ This origin can therefore be the centroid of positive masses at a suitably chosen finite and truly $m$-dimensional subset of points ${ }^{6}$

$\mathfrak{M}_{2}$ :

$$
\left(Q_{1}\left(x^{p}\right), Q_{2}\left(x^{p}\right), \cdots, Q_{m}\left(x^{p}\right)\right), \quad p=1,2, \cdots, r .
$$

To the finite set $\mathfrak{M}_{2}$ we arbitrarily adjoin the set of $n$ points $\mathfrak{M}_{3}$ :

$$
\left(Q_{1}\left(x^{j}\right), Q_{2}\left(x^{j}\right), \cdots, Q_{m}\left(x^{j}\right)\right), \quad j=1,2, \cdots, n,
$$

where each $n$-partite number $x^{j}=\left(x_{1}^{j}, x_{2}^{j}, \cdots, x_{n}^{j}\right)$ has all zero components with the exception that $x_{j}^{j}=1$.

The set $\mathfrak{N} \equiv \mathfrak{M}_{2}+\mathfrak{M}_{3}$ is a truly $m$-dimensional finite set, and its convex extension contains the origin as an inner point. Hence this origin can be the centroid of positive masses at all points of $\mathfrak{N}$; and the analytic expression of this fact leads to the system of relations

${ }^{5} \mathrm{Cf}$. Dines, Convex extension and linear equalities, Bull. Amer. Math. Soc. vol. 42 (1936) p. 357 Theorem 3.

${ }^{6}$ Ibid. p. 358.

${ }^{7}$ Ibid. p. 358 Theorem 4. 


$$
\sum_{j=1}^{n} \mu_{j} Q_{i}\left(x^{j}\right)+\sum_{p=1}^{r} \nu_{p} Q_{i}\left(x^{p}\right)=0, \quad i=1,2, \cdots, m,
$$

where each $\mu_{j}$ and $\nu_{p}$ is positive.

Applying to the relations (4) precisely the same type of argument as was applied to relations (2) in proving the lemma of $\S 2$, we arrive at the existence of a quadratic form $B(x)$ which is $c$-orthogonal to all the $Q_{i}$, which has the formal expression

$$
B(x)=\sum_{j=1}^{n} \mu_{j} x_{j}^{2}+\sum_{p=1}^{r} \nu_{p}\left(\sum_{k=1}^{n} x_{k}^{p} x_{k}\right)^{2}
$$

and is therefore certainly definite. This completes the proof of $I(b)$.

4. Proof of Theorem I(c). This follows almost immediately from $\mathrm{I}(\mathrm{a})$ and $\mathrm{I}(\mathrm{b})$. First suppose there is a $\sum \lambda_{i} Q_{i}$ which is semi-definite, but none which is definite. Then by $I(b)$ there is no definite form $c$-orthogonal to all $Q_{i}$, and so by $\mathrm{I}(\mathrm{a})$ there must be one which is semidefinite.

Conversely, suppose there exists a semi-definite (but no definite) form $c$-orthogonal to all the $Q_{i}$. Then by I(a) there exists no definite linear combination $\sum \lambda_{i} Q_{i}$, and hence by I(b) there must exist a semidefinite $\sum \lambda_{i} Q_{i}$.

5. The system of equations $\left(Q_{i} \cdot B\right)=0$. The substance of Theorem I can be equivalently expressed in terms of the system of equations

$$
\left(Q_{i} \cdot B\right)=0, \quad i=1,2, \cdots, m,
$$

where the $Q_{i}$ are given quadratic forms and $B$ is a quadratic form restricted by the system of equations.

THEOREM II. The system of equations (5)

(a) can admit only indefinite solutions $B$ if the $Q_{i}$ admit a definite linear combination,

(b) admits a definite solution $B$ if every linear combination of the $Q_{i}$ is indefinite,

(c) admits a semi-definite (but no definite) solution $B$ if the $Q_{i}$ admit a semi-definite (but no definite) linear combination.

It will be noted that the system certainly admits some solution $B$ except in the case where there exists a definite linear combination of the $Q_{i}$. In this latter case there may be indefinite solutions or no solution as illustrated in the following two examples.

EXAMPLE 1. The two forms 


$$
Q_{1}=2 x_{1}^{2}-x_{2}^{2}, \quad Q_{2}=x_{1}^{2}-2 x_{2}^{2}
$$

admit the definite linear combination $Q_{1}-Q_{2}=x_{1}^{2}+x_{2}^{2}$, and the corresponding system (5) admits the indefinite solution $B=x_{1} x_{2}$.

EXAMPLE 2. The three forms

$$
Q_{1}=2 x_{1}^{2}-\stackrel{2}{x_{2}}, \quad Q_{2}=x_{1}^{2}-2 x_{2}^{2}, \quad Q_{3}=x_{1} x_{2}
$$

admit the definite linear combination $Q_{1}-Q_{2}-Q_{3}=x_{1}^{2}-x_{1} x_{2}+x_{2}^{2}$, but the corresponding system (5) admits no solution form $B$.

Carnegie Institute of Technology

\title{
NOTE ON A CONJECTURE DUE TO EULER
}

\author{
E. T. BELL
}

Euler's conjecture (1772) that

$$
x_{1}^{n}+\cdots+x_{t}^{n}=x^{n}
$$

where $n$ is an integer greater than 3 and $2<t<n$, has no solution in rational numbers $x_{1}, \cdots, x_{t}, x$ all different from zero, is still unsettled even in its first case, $n=4, t=3$. It may therefore be of some interest to note a solution of this equation for any $n>3$ and any $t>1$ in terms of (irrational) algebraic numbers, which can be made algebraic integers by suitable choice of a homogeneity parameter, all different from zero, all the numbers being polynomials in numbers of degree $2 d$, where $4 d \leqq 2 n-5+(-1)^{n}$. If solutions differing only by a parameter are not considered distinct, there are at least $d^{t-1}$ sets of solutions $x_{1}, \cdots, x_{t}, x$.

The solutions described are

$$
\begin{gathered}
x_{1}=u, \quad x_{2}=r_{t-1} u, \quad x=\left(1+r_{1}\right) \cdots\left(1+r_{t-1}\right) u \\
x_{j}=r_{t-j+1}\left(1+r_{t-j+2}\right)\left(1+r_{t-j+3}\right) \cdots\left(1+r_{t-1}\right) u, \quad j=3, \cdots, t,
\end{gathered}
$$

where $u$ is a parameter and the $r$ 's are any roots, the same or different, of any factor $F_{n}(r)$, irreducible in the field of rational numbers, of

$$
f(r) \equiv \sum_{s=1}^{n-1}(n, s) r^{n-s-1},
$$

Received by the editors July 9, 1942. 